\title{
Calidad de vida en pacientes con esclerosis múltiple atendidos en una institución de salud de Medellín, Colombia
}

\author{
Quality of life of patients with multiple sclerosis treated at a health institution in Medellin, Colombia
}

\author{
Catalina Orozco-González ${ }^{*}$ orcid.org/0000-0001-7845-7279 \\ Basilio Vagner-Ramírez ${ }^{1}$ orcid.org/0000-0002-7957-7923 \\ Carolina Salas-Zapata ${ }^{2}$ orcid.org/0000-0002-1565-5021
}

1 Medicarte IPS. Medellín, Colombia

2 Facultad de Medicina, Universidad CES. Medellín, Colombia

Orozco-González C, Vagner-Ramírez B, Salas-Zapata C. Calidad de vida en pacientes con esclerosis múltiple atendidos en una institución de salud de Medellín, Colombia. Univ. Salud. 2019;21(3):226-234. DOI: http://dx.doi.org/10.22267/rus.192103.159

\section{Resumen}

Introducción: La calidad de vida relacionada con salud (CVRS) en la esclerosis múltiple (EM), puede verse afectada por factores físicos, clínicos y sociodemográficos. Objetivo: Determinar el efecto de factores sociodemográficos, clínicos y físicos en la calidad de vida (CV) de pacientes con EM. Materiales y métodos: Se aplicó el instrumento WHOQOL-BREF de la OMS a 173 pacientes de una institución de salud. Se realizó análisis descriptivo de características sociodemográficas, clínicas y físicas y sus puntajes de CV. Se observó la asociación entre CV global con factores sociodemográficos, clínicos y físicos; finalmente, por medio de análisis multivariado. Resultados: El 80,3\% fueron mujeres, la mediana de edad de 43 años (RIC= 51-35). La fatiga fue el síntoma más frecuente ((71\%). Los factores de riesgo para tener peor CV fueron: sexo femenino $(\mathrm{RP}=6,92$. IC 95\% 1,8-26,58), alteración en control de esfínteres ( $\mathrm{RP}=6,10$ IC 95\% 1,26-29,51), trastornos cognitivos $(\mathrm{RP}=4,46$ IC 95\% 1,07-18,56), riesgo de depresión ( $\mathrm{RP}=3,82$ IC 95\% 1,01-14,38) y no realizar fisioterapia ( $\mathrm{RP}=4,48 \mathrm{IC} 95 \%$ 1,08-18,34). Conclusiones: La afectación de CV en la EM, según factores sociodemográficos y clínicos es variable. Evaluar la CVRS en la práctica clínica, permite entender el comportamiento y necesidades del paciente para intervenir factores de riesgo.

Palabras clave: Esclerosis múltiple; calidad de vida relacionada con la salud; personas con discapacidad; actividades de la vida diaria; factores de riesgo. (Fuente: DeCS, Bireme)

\begin{abstract}
Introduction: Health-related quality of life (HRQL) in multiple sclerosis (MS) patients can be affected by physical, clinical and sociodemographic factors. Objective: To determine the effect of sociodemographic, clinical and physical factors on the quality of life (QL) of patients with MS. Materials and methods: The WHO WHOQOL-BREF instrument was applied to 173 patients of a health institution. Descriptive analysis of sociodemographic, clinical and physical characteristics and their QL scores were performed. The association between global QL with sociodemographic, clinical and physical factors was observed; finally, through multivariate analysis. Results: $80.3 \%$ were women, the median age was 43 years (IQR $=51-35$ ). Fatigue was the most frequent symptom (71\%). The risk factors for worse QL were: female sex (RP $=6.92 .95 \% \mathrm{CI} 1.8-26.58)$, abnormal sphincter control $(\mathrm{RP}=6.1095 \%$ CI 1.26-29.51), cognitive disorders ( $\mathrm{RP}=4.4695 \%$ CI 1.07-18.56), risk of depression ( $\mathrm{RP}=3.82$ 95\% CI 1.01-14.38) and lack of physiotherapy ( $\mathrm{RP}=4.4895 \% \mathrm{CI} 1.08-18.34)$. Conclusions: The effect of QL in MS is variable and depends on sociodemographic and clinical factors. Evaluating the (HRQL) in clinical practice facilitates the understanding of the behavior and needs of the patient required for risk factors intervention.
\end{abstract}

Key words: Multiple sclerosis; health related quality of life; disabled persons; activities of daily living; risk factors. (Source: DeCS, Bireme). 


\section{Introducción}

La esclerosis múltiple (EM) es una enfermedad crónica, degenerativa y autoinmune del sistema nervioso central que afecta aproximadamente dos millones de personas en todo el mundo; se caracteriza por inicio entre los 20-40 años de edad. Dentro de sus principales síntomas se encuentran el compromiso visual, la alteración sensorial, compromiso motor, fatiga y trastorno depresivo y cognitivo. La EM tiene un curso impredecible, al igual que su gravedad y pronóstico; existen 3 formas de la EM: a) EM remitente- recurrente (EMRR): en la que ocurren brotes con duración variable, con recuperación total o parcial de los síntomas en días o meses, afecta aproximadamente el $85 \%$ de la población; b) EM secundaria progresiva (EMSP): inicia como EMRR y empeora progresivamente con el tiempo, con o sin presencia de brotes; c) EM primaria progresiva (EMPP): inicio lento y empeoramiento progresivo de síntomas de manera constante, sin brotes, afecta el $10 \%$ de los individuos ${ }^{(1,2)}$.

El tratamiento de la EM tiene tres categorías: manejo de recaídas, terapias modificadoras de la enfermedad y manejo sintomático. El objetivo del tratamiento es disminuir la velocidad de progresión de la discapacidad y frecuencia de recaídas. Las terapias modificadoras de la enfermedad se dividen en moderada eficacia o terapias de inicio, entre los que se encuentran interferón beta $1 \mathrm{~A}$ y $1 \mathrm{~B}$ en sus distintas formas de aplicación, acetato de glatiramero, teriflunomida y dimetilfumarato; y terapias de alta eficacia o segunda línea: fingolimod, natalizumab, alemtuzumab, y el ocrelizumab, de reciente aprobación y único con indicación para formas primarias progresivas. La fampridina es un medicamento coadyuvante indicado para mejorar la marcha en los pacientes con EM con EDSS entre 3 y $7^{(3-6) .}$

El deterioro de la calidad de vida relacionada con salud (CVRS), se ha asociado a limitaciones funcionales, compromiso cognitivo, y problemas emocionales( $(7,8)$; esto, a su vez se asocia con un costo indirecto de productividad, resultado del incremento de los costos públicos en términos de pensiones por ausentismo laboral temprano, discapacidad $\mathrm{y}$ jubilaciones anticipadas ${ }^{(9-11)}$.

En los últimos años, dentro del manejo integral de las personas con EM, se ha incluido la realización de una valoración de la CVRS, que brinda información sobre las necesidades del paciente o complicaciones no detectadas muchas veces por el equipo de salud, que es un elemento clave para evaluar el tratamiento y la necesidad de intervenciones adicionales(7,8,12). Los instrumentos para medir calidad de vida (CV) pueden ser genéricos, usados para comparar poblaciones y analizar el comportamiento de enfermedades, o específicos, que incluyen aspectos de acuerdo a las características clínicas de la patología y sus resultados tienen mejor capacidad de predicción y discriminación para evaluar cambios y efectos a través del tiempo ${ }^{(7,13-16)}$.

Aún existen pocos estudios de CV en América Latina, en Panamá, se encontró que las dimensiones de la movilidad y las actividades habituales de la vida fueron las más afectadas por la progresión de la discapacidad, mientras que la ansiedad/depresión afectaba en todas las etapas de la enfermedad(17). La población de Argentina se ha comparado en múltiples estudios con la población europea, principalmente española, y se encontró que los principales determinantes de una disminución en la CVRS se asociaron con variables clínicas (estado de discapacidad, duración de la enfermedad y síntomas como fatiga $\mathrm{y}$ depresión), $\mathrm{y}$ variables sociodemográficas (edad, sexo) ${ }^{(1,7) .}$

Este estudio tuvo como objetivo estudiar el efecto de los factores sociodemográficos, clínicos y físicos sobre la calidad de vida de los pacientes con esclerosis múltiple atendidos en una institución de salud de Medellín, Colombia.

\section{Materiales y métodos}

Se realizó un estudio transversal con intención analítica de 173 pacientes con EM que asistieron a Medicarte Medellín, durante diciembre de 2016 a febrero de 2017. Se aplicó el instrumento WHOQOLBREF de la Organización Mundial de la Salud, validado en población latinoamericana adulta, en la década de 1990-1999, para obtener una definición consensuada de calidad de vida y que pudiera aplicarse transculturalmente en población general o con problemas de salud; la versión inicial fue el WHOQOL-100, posteriormente se seleccionó la mejor pregunta de cada faceta, dando como resultado el WHOQOL-BREF(18); que consta de 26 preguntas, por cada una de las 24 facetas que lo comprenden, y dos preguntas generales que se evalúan de forma 
separada: CV global y satisfacción con la salud general; con opciones de respuesta tipo Likert, que dan como resultado un perfil de 4 dimensiones o dominios: salud física, salud psicológica, relaciones sociales y entorno/ambiente; resultado que se extrapola a la escala completa. La Escala Ampliada de Estado de Discapacidad- EDSS (Expanded Disability Status Scale), mide de acuerdo a siete grupos funcionales, el grado de disfunción de pacientes desde cero que indica normalidad, hasta diez que indica muerte por esclerosis múltiple(19).

Para evaluar el riesgo de depresión se aplicó la escala de Zung, un instrumento de 20 ítems que indaga por 4 características comunes de depresión: el efecto dominante, equivalentes fisiológicos, otras perturbaciones, y actividades psicomotoras; tiene diez preguntas elaboradas de forma positiva, y otras diez, de forma negativa; cada ítem presenta cuatro opciones de respuesta (valoradas de uno a cuatro puntos); la valoración total oscila entre 20 y 80 , con punto de corte significativo para síntomas depresivos de 40; así, a mayor puntuación, mayor severidad del síntoma que sugiere depresión. Finalmente, los valores se convierten multiplicando por 1,25; así las puntuaciones que informan se encuentran entre $25 \mathrm{y}$ 100 puntos(20-23). Se realizó una prueba piloto donde se escogieron 10 pacientes al azar, con los cuales se probó la metodología, se ajustaron las preguntas, la duración del tiempo de la encuesta, se hicieron los ajustes pertinentes al cuestionario y se entrenó el coinvestigador para el diligenciamiento con cada paciente.

La información fue complementada tomando registros de la historia clínica o durante la consulta con neurología o medicina general, se indagó sobre variables sociodemográficas como: edad, sexo, estado civil, cambios en estado civil por causa de la enfermedad, persona con quien vive, máximo nivel de escolaridad, situación laboral, rendimiento laboral por la enfermedad (medido como: igual; disminuido por incremento de cansancio; disminución en número de horas; afectación grave: cambio de trabajo o deserción laboral), presencia de dificultades en las relaciones laborales con sus compañeros debido al diagnóstico, ayuda económica a causa de la EM. Sobre variables clínicas como: tiempo evolución de la enfermedad, tiempo de inicio del primer tratamiento, medicamento actual, número de recaídas en el último año, manifestaciones clínicas actuales, uso de medicamentos para manejo sintomático, presencia de comorbilidades (hipertensión arterial, diabetes mellitus, hipotiroidismo o dislipidemia, índice de masa corporal); y físicas como: requerimiento de algún tipo de apoyo para la marcha (ninguno, bastón, caminador, silla de ruedas), realización de fisioterapia en el momento del estudio y número de sesiones por semana; el cuestionario de CV fue realizado por el investigador principal.

Participaron los pacientes que cumplían criterios diagnósticos de Mc Donald 2010 (que incluye el número de episodios de brote, signos de diseminación en tiempo y espacio de lesiones en imágenes de resonancia magnética contrastada, y demostración de bandas oligoclonales en líquido cefalorraquídeo) ${ }^{(24,25)}$ y dieron su consentimiento para participar; se excluyeron pacientes menores de 18 años y en quienes se descartó EM durante el período de recolección de datos, o con otra enfermedad neurológica asociada y escala EDSS mayor de 8,5; por su mayor compromiso cognitivo y físico. En cuanto al control de calidad, se realizó procedimiento de verificación a una muestra aleatoria del $10 \%$ de encuestas digitadas, además de doble digitación y verificación de la base de datos.

Se realizó análisis descriptivo de variables sociodemográficas, clínicas y físicas, además de los puntajes para cada dominio del cuestionario WHOQOL-BREF. De acuerdo a la distribución paramétrica o no de las variables, éstas, se expresaron como media y desviación estándar (DE) o mediana y rango intercuartílico (RIC). Se analizó la asociación de factores sociodemográficos, clínicos y físicos con la $\mathrm{CV}$ global, definida como peor y mejor $\mathrm{CV}$, por medio de la prueba estadística Chi cuadrado. Se construyó un modelo de regresión logística multivariada binaria con el método hacia adelante Wald, en el cual se tuvo como variable dependiente la $\mathrm{CV}$, para este fue necesario dicotomizar esta variable, puesto que su valor original era el de puntaje de CV global, obtenido en una escala de 1 a 5 . Se calculó la Razón de Prevalencia (RP) ajustado para cada variable. Con respecto a las pruebas de hipótesis se utilizó un nivel de confianza del 95\%, con un valor de error alfa de $5 \%$. Los análisis estadísticos se realizaron utilizando el software SPSS versión 21.

\section{Consideraciones éticas}

Esta investigación se basó en el Artículo 11 de la Resolución 8430 de 1993 del Ministerio de Salud de Colombia, con un riesgo mínimo. Todos los 
participantes firmaron consentimiento informado. Se obtuvo aprobación por el Comité de Ética de la Universidad CES y de Medicarte S.A. y fue financiado con recursos propios.

\section{Resultados}

De 173 pacientes, el 80,3\% correspondía a mujeres, con una mediana de edad de 43 años (RIC= 35- 51 años), la edad mínima fue 20 años y la máxima 74 , se observó una relación mujer: hombre de 4:1. El 39,3\% eran solteros, $38,2 \%$ casados. El máximo nivel educativo de los pacientes fue universitario 45,6\%, técnico o tecnológico 27,7\%. El 26,5\% vivía con su cónyuge e hijos, el $24,9 \%$ con los padres, y el 9,9\% vivía solo. El 35,8\% conservaba su empleo, 28,9\% estaba pensionado a causa de la enfermedad, $14 \%$ trabajaba independiente, $13,3 \%$ se desempeñaba como ama de casa. De los pacientes que aún conservaban su empleo, el 24,3\% reportaron tener el mismo rendimiento laboral a pesar de la enfermedad, para el $30 \%$ el rendimiento había disminuido un poco y se cansaba más fácilmente, aproximadamente el $17 \%$ manifestó que su rendimiento se afectó gravemente y tuvieron que cambiar de trabajo o dejar de trabajar (Tabla 1).

Tabla 1. Factores sociodemográficos, clínicos y físicos asociados a la calidad de vida de los pacientes con esclerosis múltiple atendidos

\begin{tabular}{|c|c|c|c|c|c|c|}
\hline \multirow{3}{*}{ Variables } & \multicolumn{4}{|c|}{ Calidad de vida } & \multirow{3}{*}{$\begin{array}{c}\text { Valor } p \\
\mathrm{X}^{2}\end{array}$} & \multirow{3}{*}{ RP (IC 95\%) } \\
\hline & \multicolumn{2}{|c|}{ Peor } & \multicolumn{2}{|c|}{ Mejor } & & \\
\hline & $\mathbf{n}$ & $\%$ & $\mathbf{n}$ & $\%$ & & \\
\hline \multicolumn{7}{|l|}{ Sexo } \\
\hline Mujer & 10 & 55,6 & 129 & 83 & $<0,0001$ & 1 \\
\hline Hombre & 8 & 44,4 & 26 & 17 & & $0,30(0,13-0,71)$ \\
\hline \multicolumn{7}{|l|}{ Máximo nivel de escolaridad } \\
\hline Universidad/ Postgrado & 6 & 33,2 & 73 & 47,3 & 0,05 & 1 \\
\hline Primaria & 4 & 22,2 & 8 & 5,2 & & $4,38(1,44-13,31)$ \\
\hline Secundaria & 4 & 22,2 & 30 & 19,2 & & $1,54(0,46-5,14)$ \\
\hline Técnica/tecnología & 4 & 22,2 & 44 & 28,3 & & $1,09(0,32-3,69)$ \\
\hline \multicolumn{7}{|l|}{ Cambios en el rendimiento laboral de las personas que trabajan } \\
\hline Tiene el mismo rendimiento & 2 & 12,5 & 40 & 35,1 & $<0,0001$ & 1 \\
\hline Ha disminuido un poco, se cansa más fácil & 4 & 25 & 48 & 42,2 & & $1,61(0,31-8,39)$ \\
\hline Ha disminuido mucho, redujo el número de horas & 1 & 11,1 & 6 & 5,2 & & $3,00(0,31-28,84)$ \\
\hline $\begin{array}{l}\text { Gravemente afectado, he tenido que cambiar de trabajo o dejar de } \\
\text { trabajar }\end{array}$ & 9 & 56,2 & 20 & 17,5 & & $6,51(1,51-27,98)$ \\
\hline \multicolumn{7}{|l|}{ Recibe ayuda económica de la enfermedad } \\
\hline $\mathrm{Si}$ & 4 & 22,2 & 8 & 5,5 & 0,02 & 1 \\
\hline No & 14 & 77,8 & 147 & 95,4 & & $3,83(1,49-9,85)$ \\
\hline \multicolumn{7}{|l|}{ Tipo de esclerosis múltiple } \\
\hline Recaída Remisión & 11 & 61 & 131 & 84,5 & 0,03 & 1 \\
\hline Primaria Progresiva & 2 & 11 & 4 & 2,6 & & $4,30(1,21-15,26)$ \\
\hline Secundaria progresiva & 5 & 28 & 20 & 12,9 & & $2,58(0,98-6,79)$ \\
\hline \multicolumn{7}{|l|}{ Usa medicamentos para el dolor } \\
\hline No & 6 & 33 & 96 & 61,9 & 0,02 & 1 \\
\hline $\mathrm{Si}$ & 12 & 67 & 59 & 38,1 & & $1,72(0,83-3,56)$ \\
\hline \multicolumn{7}{|l|}{ Usa medicamentos para la espasticidad } \\
\hline No & 13 & 72 & 140 & 90,3 & 0,03 & 1 \\
\hline $\mathrm{Si}$ & 5 & 28 & 15 & 9,7 & & $2,87(1,18-6,96)$ \\
\hline \multicolumn{7}{|l|}{ Síntomas sensitivos } \\
\hline No & 4 & 22 & 75 & 48,4 & 0,04 & 1 \\
\hline $\mathrm{Si}$ & 14 & 78 & 80 & 51,6 & & $1,50(1,12-2,01)$ \\
\hline \multicolumn{7}{|l|}{ Síntomas motores } \\
\hline No & 5 & 28 & 98 & 63,2 & 0,005 & 1 \\
\hline $\mathrm{Si}$ & 13 & 72 & 57 & 36,8 & & $1,96(1,37-2,79)$ \\
\hline \multicolumn{7}{|l|}{ Síntomas del tronco encefálico } \\
\hline No & 9 & 50 & 116 & 74,8 & 0,04 & 1 \\
\hline $\mathrm{Si}$ & 9 & 50 & 39 & 25,2 & & $1,98(1,16-3,39)$ \\
\hline
\end{tabular}




\begin{tabular}{|c|c|c|c|c|c|c|}
\hline \multicolumn{7}{|l|}{ Fatiga } \\
\hline No & 1 & 5,6 & 48 & 31 & 0,02 & 1 \\
\hline $\mathrm{Si}$ & 17 & 94,4 & 107 & 69 & & $2,28(1,83-2,84)$ \\
\hline \multicolumn{7}{|c|}{ Alteración en control de esfínteres } \\
\hline No & 3 & 17 & 91 & 58,7 & 0,001 & 1 \\
\hline $\mathrm{Si}$ & 15 & 83 & 64 & 41,3 & & $2,01(1,52-2,66)$ \\
\hline \multicolumn{7}{|c|}{ Trastornos cognitivos } \\
\hline No & 5 & 28 & 107 & 69 & 0,001 & 1 \\
\hline $\mathrm{Si}$ & 13 & 72 & 48 & 31 & & $2,32(1,60-3,37)$ \\
\hline \multicolumn{7}{|c|}{ Riesgo de depresión } \\
\hline No & 6 & 33 & 10 & 6,5 & 0,02 & 1 \\
\hline $\mathrm{Si}$ & 12 & 67 & 145 & 93,5 & & $0,71(0,51-0,99)$ \\
\hline \multicolumn{7}{|c|}{ Actualmente realiza fisioterapia } \\
\hline $\mathrm{Si}$ & 3 & 16,7 & 51 & 33 & 0,18 & 1 \\
\hline No & 15 & 83,3 & 104 & 67 & & $0,50(0,17-1,45)$ \\
\hline
\end{tabular}

La EMRR tuvo una prevalencia del 82,1\%, EMSP del 14,5\% y EMPP del 3,5\%; el 73,4\% no presentaron ninguna recaída en el último año. Se encontró que $11,6 \%$ no utilizaban ningún medicamento inmunomodulador por decisión propia. Dentro de las manifestaciones clínicas que presentaron los pacientes en el último mes, los más frecuentes fueron la fatiga (71,7\%) y los síntomas sensitivos (54,3\%), el 91\% de los pacientes tenían riesgo de depresión; se presentaron síntomas motores en el 40,5\%, síntomas del tronco encefálico en el $27 \%$, alteraciones visuales $31,8 \%$, dolor $37 \%$, alteración en el control de esfínteres 45,7\%, trastornos cognitivos 35,3\% y alteraciones sexuales en 19,7\%. Además del medicamento inmunomodulador (figura 1), el $42 \%$ utilizaba medicamentos para dolor, 34,1\% antidepresivos, 20,8\% medicamentos para la fatiga, $11,6 \%$ medicamentos para espasticidad, $8,7 \%$ usaba fampridina para mejorar la marcha y $8,1 \%$ medicamentos para manejo de síntomas urinarios.

Figura 1. Distribución porcentual del tratamiento actual de los pacientes en Medicarte, Medellín durante 2017

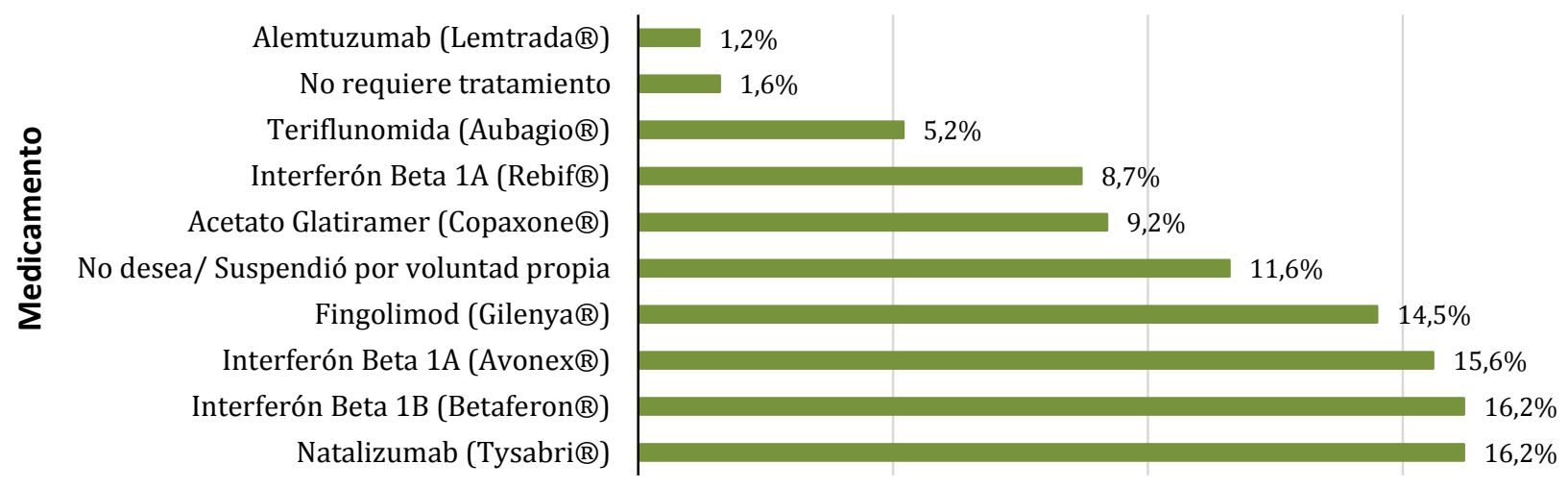

El 14,5\% presentaron hipertensión arterial, el 11,6\% hipotiroidismo, el 10,4\% dislipidemia y se presentó diabetes mellitus en el 4,6\%. De acuerdo a la escala EDSS, el $66,5 \%$ estaba entre 0 y 2 puntos y el $5,8 \%$ tuvo 8,0 . El $67 \%$ de los pacientes no requería ningún tipo de apoyo para el desplazamiento y el 31,2\% realizaba fisioterapia. Al analizar el cuestionario WHOQOL-BREF, se encontró que la CV global obtuvo una mediana de 4 (bastante buena) y para la satisfacción con la salud se obtuvo una mediana de 3 (lo normal), además que el dominio físico fue el más afectado (Tabla 2).

Se construyó una variable dependiente de calidad de vida categorizada como " $0=$ menos de 3 puntos" $\mathrm{y}$ " $1=$ mayor o igual a 3 puntos", de acuerdo al cálculo realizado previamente del primer quintil, donde el $20 \%$ de los pacientes encuestados tuvieron una 
calificación de 3 o menos (puntajes más bajos), es decir peor calidad de vida.

Para los factores sociodemográficos, por medio de la prueba Chi cuadrado de independencia, se encontró asociación entre CV y: sexo, nivel de escolaridad, cambios en el rendimiento laboral, el hecho de recibir ayuda económica debido a la enfermedad.
Para los factores clínicos, se encontró asociación entre $\mathrm{CV}$ y pacientes con EM tipo primaria progresiva, con síntomas sensitivos, síntomas motores, síntomas del tronco encefálico, fatiga, alteración en el control de esfínteres, trastornos cognitivos, con riesgo de depresión, uso de medicamentos para el dolor, y para la espasticidad; variables que se incluyeron en el modelo de regresión logística (Tabla 1).

Tabla 2. Indicadores de resumen de dominios de calidad de vida del WHOQOL-BREF

\begin{tabular}{lccccc}
\hline \multicolumn{1}{c}{ Variable } & Mediana & DE & Mediana & $\begin{array}{c}\text { Rango IC (Q3- } \\
\text { Q1) }\end{array}$ & $\begin{array}{c}\text { Coeficiente } \\
\text { variación }\end{array}$ \\
\hline Puntaje calidad de vida general* $^{*}$ & 3,61 & 0,94 & 4 & $4,0-3,0$ & 26,03 \\
Puntaje satisfacción con la salud* $_{\text {Puntaje dominio físico** }}^{3,31}$ & 1,06 & 3 & $4,0-3,0$ & 32,02 \\
Puntaje dominio psicológico** $^{* *}$ & 45,99 & 17,51 & 44 & $56-34,5$ & 38,07 \\
Puntaje dominio social** $_{\text {Puntaje dominio entorno** }}^{* 6,65}$ & 17,7 & 69 & $81-50$ & 26,96 \\
\hline
\end{tabular}

*Escala 1 a 5 ** Escala de 0 a 100

Finalmente, en al análisis de regresión logística multivariada, se encontró que ser mujer, tener alteración en el control de esfínteres, presentar trastornos cognitivos, tener riesgo depresión y la no realización de fisioterapia, fueron las características que en conjunto explicaron el $37 \%$ del impacto negativo en la CV, al ajustar por los demás factores que se incluyeron en el modelo, factores que además se comportaron como factores de riesgo para afectar la CV de los pacientes con esclerosis múltiple al ajustar por los demás factores, pero estos RP tan altos, con IC 95\% muy amplios indican una estimación poco precisa, probablemente debido al tamaño de la muestra (Tabla 3).

Tabla 3. Regresión logística multivariada para la calidad de vida global según algunas características de los pacientes con esclerosis múltiple

\begin{tabular}{|c|c|c|c|c|c|c|}
\hline Variable & B & Error estándar & Wald & Valor p & $\mathbf{R P}$ & IC $95 \%$ \\
\hline Sexo & $-1,93$ & 0,68 & 7,93 & 0,005 & 6,92 & $1,8-26,58$ \\
\hline Alteración en control de esfínteres & 1,81 & 0,80 & 5,07 & 0,020 & 6,10 & $1,26-29,51$ \\
\hline Trastorno cognitivo & 1,49 & 0,72 & 4,22 & 0,040 & 4,46 & $1,07-18,57$ \\
\hline Riesgo de depresión & 1,33 & 0,67 & 3,89 & 0,040 & 3,82 & $1,01-14,38$ \\
\hline Actualmente realiza fisioterapia & 1,49 & 0,72 & 4,28 & 0,030 & 4,48 & $1,08-18,34$ \\
\hline Constante & 2,76 & 0,70 & 15,59 & 0,000 & 15,91 & \\
\hline
\end{tabular}

Categoría de referencia: ${ }^{*}$ sexo: femenino ${ }^{* *}$ No alteración en control de esfínteres ${ }^{* * *}$ No trastorno cognitivo ${ }^{* * * *}$ No riesgo de depresión ${ }^{* * * * *}$ Actualmente realiza fisioterapia

\section{Discusión}

La EM genera cambios inflamatorios y degenerativos que producen una variedad de síntomas que comprometen el autocuidado e independencia de los pacientes y puede asociarse a trastornos depresivos, afectando las relaciones sociales, laborales y familiares(17,26). El 29\% de los pacientes estaban pensionados por la enfermedad, asociado a deserción laboral por múltiples causas, dentro de las más importantes el incremento en la discapacidad y secuelas físicas que comprometieron el rendimiento laboral. Según datos del Global MS Employment Report de 2016, el 43\% de las personas dejaron de trabajar a los tres años del diagnóstico, con un incremento del $70 \%$ a los 10 años(27); por esta razón se deben estudiar las causas de deserción laboral de esta población para desarrollar políticas incluyentes que prevengan la pérdida de capacidad laboral temprana y disminuyan costos públicos en términos de pensiones por discapacidad. 
La fatiga, descrita como una sensación de agotamiento para realizar actividades físicas $o$ intelectuales, desproporcional al tipo de esfuerzo que se realiza y al grado de discapacidad que se presenta; es uno de los síntomas más frecuentes en la población con EM, afecta casi el 80\% de los pacientes, se asocia a empeoramiento de otros síntomas, limitación de las funciones físicas y laborales de los pacientes, y en algunos casos es la principal causa de deserción laboral. La fatiga y la alteración en el estado de ánimo se han relacionado con alteración del funcionamiento cognitivo de las personas con $\operatorname{EM}(7,13,28,29)$. La presencia de síntomas sensitivos y fatiga, generan un impacto negativo en actividades de la vida cotidiana, dado por la disminución en la sensación de vitalidad durante el día y empeoramiento de la función física, lo que se refleja en el deterioro de la CV e incremento de ausentismo laboral(8,12,30-32).

Se encontró que el 90\% de los pacientes del estudio tuvieron riesgo leve o moderado de depresión evaluado con el test de Zung. De acuerdo con Olascoaga(13), la presencia de factores psicológicos afecta de manera negativa la $\mathrm{CV}$, por incremento en niveles de fatiga, disfunción física, incremento de dolor, alteraciones cognitivas y trastornos del sueño, que pueden reflejarse la falta de adherencia al tratamiento y deterioro $\mathrm{CV}$ de las personas con EM, que podría desencadenar un peor pronóstico(12,32-36).

En la población de estudio, se encontró que no realizar fisioterapia fue un factor de riesgo para tener peor CV; la evidencia ha demostrado que la actividad física en EM es una intervención no farmacológica que tiene efectos beneficiosos en la capacidad funcional, cognitiva y debe incluirse dentro del tratamiento de las personas con EM(28,37-41). No se interrogaron las causas para no realizar fisioterapia, se debe estudiar si se debe a barreras de acceso a servicios de salud $u$ otras causas.

La diferencia en los puntajes de las dimensiones de la CV puede deberse a la diversidad de presentación de las manifestaciones clínicas en cada paciente y su percepción en el impacto en la CV, lo que afecta de manera diferente cada dimensión, aunque se encontró que la dimensión física fue la más afectada; la combinación de escalas genéricas y específicas brindan una mejor información sobre los síntomas de la EM y su impacto en la CV. En el estudio de validación del cuestionario de calidad de vida internacional de esclerosis múltiple (MusiQoL), se encontró que el bajo nivel educativo, alto EDSS, el compromiso cognitivo, ser soltero, fueron los factores que afectaron la puntuación global del cuestionario, mientras que ser mujer, tener mayor edad, mayor EDSS afectaban más el dominio físico del cuestionario de $C V$, lo que evidencia que a pesar de la variedad de síntomas de la EM, los factores que más afectan la CV tienen un comportamiento similar en todos los pacientes $(7,8,12,14-16)$.

Dado que la muestra fue a conveniencia por la forma de la atención de los pacientes, no es posible inferir los resultados a todos los pacientes con esclerosis múltiple ni atribuir causalidad a los factores encontrados como asociados a la CV. El instrumento de CVRS empleado fue genérico por cuanto no se contaba con uno específico en el momento del estudio por dificultades con la licencia, tampoco se utilizaron cuestionarios específicos para evaluar la fatiga. La CV puede estar afectada por otros aspectos no incluidos en este estudio como red de apoyo, acceso a servicios de salud, capacidad económica, etc. Respecto a la presencia de comorbilidades, no se indagó sobre criterios diagnósticos para definir la presencia de comorbilidades, o si se trataba de efectos adversos por el uso de medicamentos.

\section{Conclusiones}

Se observó que los principales factores de riesgo para tener un mayor compromiso en la calidad de vida en pacientes con esclerosis múltiple fueron ser mujer, tener alteración en el control de esfínteres, presentar trastornos cognitivos, tener riesgo depresión y la no realización de fisioterapia. LA EM tiene un curso impredecible, por lo que el grado de afectación en la CV de acuerdo a factores sociodemográficos y clínicos es diferente en cada persona.

\section{Recomendaciones}

Se debe incluir la fatiga dentro de futuros estudios y desarrollar medidas encaminadas a disminuir los niveles de esta, para que el paciente pueda mejorar su calidad de vida y rendimiento laboral. Realizar evaluación de la CVRS dentro de la práctica clínica diaria, permite tener un mejor entendimiento de las necesidades insatisfechas de esta población, realizar intervención de factores susceptibles (capacidad funcional y cognitiva), y barreras de acceso al sistema de salud, que facilitan el desarrollo de programas de atención integral, incluyendo medidas no farmacológicas, como el acompañamiento psicológico 
y la terapia física, los cuales han mostrado gran beneficio en la capacidad funcional, cognitiva y en la CV de las personas con EM.

\section{Agradecimientos}

Los autores agradecen de manera especial a la EPS Sura y a los pacientes por todo el apoyo en la realización de este estudio.

\section{Conflicto de intereses}

Los autores declaran que no tienen ningún conflicto de interés.

\section{Referencias}

1. Kidd T, Carey N, Mold F. A systematic review of the effectiveness of self-management interventions in people with multiple sclerosis at improving depression, anxiety and quality of life. PLoS One. 2017;12(10):e0185931.

2. Browne P, Chandraratna D, Angood C. Atlas of Multiple Sclerosis 2013: A growing global problem with widespread inequity. Neurology. 2014;83(11):1022-4.

3. Montalban X, Gold R, Thompson AJ. ECTRIMS/EAN Guideline on the pharmacological treatment of people with multiple sclerosis. Mult Scler J. 2018;24(2):96-120.

4. Hart FM, Bainbridge J. Current and emerging treatment of multiple sclerosis. Am J Manag Care. 2016;22(6):159-70.

5. Vargas DL, Tyor WR. Update on disease-modifying therapies for multiple sclerosis. J Investig Med. 2017;65(5):883-91.

6. Vargas DL, Tyor WR. Update on disease-modifying therapies for multiple sclerosis. J Investig Med. 2017;65(5):883-91.

7. Fernández 0, Baumstarck-Barrau K, Simeoni MC. Patient characteristics and determinants of quality of life in an international population with multiple sclerosis: Assessment using the MusiQoL and SF-36 questionnaires. Mult Scler J. 2011;17(10):1238-49.

8. Baumstarck K, Pelletier J, Butzkueven H. Health-related quality of life as an independent predictor of long-term disability for patients with relapsing-remitting multiple sclerosis. Eur J Neurol. 2013;20(6):907-14.

9. Ivanova JI, Birnbaum HG, Samuels S, Davis M, Phillips AL, Meletiche D. The cost of disability and medically related absenteeism among employees with multiple sclerosis in the US. Pharmacoeconomics. 2009;27(8):681-91.

10. Cores E V, Vanotti S, Burin DI. Factores asociados con la situación laboral de pacientes con esclerosis múltiple TT Factors associated to the work situation of patients with multiple sclerosis. Rev Neurol. 2014;58(4):175-83.

11. Patti F. Effects of education level and employment status on HRQoL in early relapsing-remitting multiple sclerosis. Mult Scler. 2007;13(6):783-91.

12. Benedict RH, Wahlig E, Bakshi R, Fishman I, Munschauer F, Zivadinov R, et al. Predicting quality of life in multiple sclerosis: accounting for physical disability, fatigue, cognition, mood disorder, personality, and behavior change. J Neurol Sci. 2005;231(1-2):29-34.

13. Olascoaga J. Calidad de vida y esclerosis múltiple. Rev Neurol. 2010;51(5):279-88.
14. Simeoni MC, Auquier P, Fernandez O, et al. Validation of the Multiple Sclerosis International. Mult Scler. 2008;14(2007):219-30.

15. Fernández 0 , Fernández V, Baumstarck-Barrau. Validation of the spanish version of the multiple sclerosis international quality of life (musiqol) questionnaire. BMC Neurol. 2011;11(1):127.

16. Boucher F, Baumstarck K, Acquadro C. Challenges In Translating The Multiple Sclerosis International Quality of Life (Musiqol) Questionnaire In 57 Languages. Value Heal. 2015;18(7):A762.

17. Gracia F, Larreategui M, Rodríguez G. Costs of multiple sclerosis in Panama from societal, patient perspectives and healthrelated quality of life. PLoS One. 2018;13(10):1-12.

18. WHOQOL. Development of the WHOQOL: Rationale and Current Status. Int J Ment Health. 2014;23(3):24-56.

19. Kurtzke JF. Rating neurologic impairment in multiple sclerosis: An expanded disability status scale (EDSS). Neurology. 1983;33(11):1444-1444.

20. William WK, Zung MD. A self rating depression scale. Arch gen psych. 1965;12:63-70.

21. William WK. Factors influencing the Self-Rating Depression Scale. Arch Gen Psychiat. 1967;16:543-7.

22. Campo-Arias A. Comparación de algunas propiedades psicométricas de la escala de Zung para depresión con puntuaciones dicotómicas. Rev Colomb Psiquiatr. 2006;35(4):511-8.

23. Campo-Arias A, Díaz-Martínez LA, Rueda-Jaimes GE. Validación de la escala de Zung para depresión en universitarias de Bucaramanga, Colombia Validation of Zung's Self-Rating Depression Scale Among Universitary Student Women from Bucaramanga, Colombia. Rev Colomb Psiquiatr. 2005;34(1):54-62.

24. Polman $\mathrm{CH}$, Reingold SC. Diagnostic criteria for multiple sclerosis: 2010 Revisions to the McDonald criteria. Ann Neurol. 2011;69(2):292-302.

25. Alan J Thompson, Brenda L Banwell FB. Diagnosis of multiple sclerosis: revision of the McDonald criteria 2017. Lancet Neurol. 2018;89(17):162-73.

26. Salter AR, Cutter GR, Tyry T. Impact of loss of mobility on instrumental activities of daily living and socioeconomic status in patients with MS. Curr Med Res Opin. 2010;26(2):493-500.

27. MS International Federation. Informe mundial sobre el empleo y la EM 2016. London: MS; 2016.

28. Smith C, Hale L, Olson K. How does exercise influence fatigue in people with multiple sclerosis. Disabil Rehabil. 2009;31(9):685-92.

29. Freeman JA, Hendrie W, Creanor S. Standing up in multiple sclerosis (SUMS): Protocol for a multi-centre randomised controlled trial evaluating the clinical and cost effectiveness of a home-based self-management standing frame programme in people with progressive multiple sclerosis. BMC Neurol. 2016;16(1):1-10.

30. Benito-León J, Mitchell AJ, Rivera-Navarro J. Impaired healthrelated quality of life predicts progression of disability in multiple sclerosis. Eur J Neurol. 2013;20(1):79-86.

31. Wilski M, Tasiemski T. Health-related quality of life in multiple sclerosis: role of cognitive appraisals of self, illness and treatment. Qual Life Res. 2016;25(7):1761-70.

32. Yalachkov Y, Soydaş D, Bergmann J. Determinants of quality of life in relapsing-remitting and progressive multiple sclerosis. Mult Scler Relat Disord. 2019;30(January):33-7. 
33. Wang Y, Gorenstein C. Assessment of depression in medical patients: A systematic review of the utility of the Beck Depression Inventory-II. Clinics. 2013;68(9):1274-87.

34. Tepavčević DK, Pekmezović T, Drulović J. Quality of life assessment in patients with multiple sclerosis | Ispitivanje kvaliteta života bolesnika sa multiplom sklerozom. Vojnosanit Pregl. 2009;66(8):645-51.

35. Arnett P, Ben-Zacharia A, Benedict R. The Goldman Consensus statement on depression in multiple sclerosis. Mult Scler. 2005;11(3):328-37.

36. Sánchez-López MP, Olivares-Pérez T, Nieto-Barco A. Esclerosis múltiple y depresión. Rev Neurol. 2004;38(6):524-9.

37. Hale LA, Smith C, Mulligan H. Tell me what you want, what you really really want: Asking people with multiple sclerosis about enhancing their participation in physical activity. Disabil Rehabil. 2012;34(22):1887-93.

38. Tabrizi FM, Radfar M. Fatigue, sleep quality, and disability in relation to quality of life in multiple sclerosis. Int J MS Care. 2015;17(6):268-74.

39. Virtanen A, Ruutiainen J, Aunola S. Effects of a 6-month exercise program on patients with multiple sclerosis: a randomized study. Neurology. 2004;63(11):2034-8.

40. Halabchi F, Alizadeh Z, Sahraian MA. Exercise prescription for patients with multiple sclerosis; potential benefits and practical recommendations. BMC Neurol. 2017;17(1):1-11.

41. Dalgas U. Exercise therapy in multiple sclerosis and its effects on function and the brain. Neurodegener Dis Manag. 2017;7(6):35-40. 